\title{
A Proposed Reductionist Solution to Address the Methodological Challenges of Inconsistent Reflexology Maps and Poor Experimental Controls in Reflexology Research: A Discussion Paper
}

\author{
Jenny Jones, BSc (Hons), RN,' Patricia Thomson, PhD, MPH, MA, DipN, RN, RNT,2 \\ William Lauder, PhD, RMN, ${ }^{2}$ and Stephen J. Leslie, BSc, MB, ChB, FRCP, PhD ${ }^{1,3}$
}

\begin{abstract}
Reflexology is a complex massage intervention, based on the concept that specific areas of the feet (reflex points) correspond to individual internal organs within the body. Reflexologists trained in the popular Ingham reflexology method claim that massage to these points, using massage techniques unique to reflexology, stimulates an increase in blood supply to the corresponding organ. Reflexology researchers face two key methodological challenges that need to be addressed if a specific treatment-related hemodynamic effect is to be scientifically demonstrated. The first is the problem of inconsistent reflexology foot maps; the second is the issue of poor experimental controls. This article proposes a potential experimental solution that we believe can address both methodological challenges and in doing so, allow any specific hemodynamic treatment effect unique to reflexology to experimentally reveal itself.
\end{abstract}

\section{Introduction}

$\mathbf{R}$ EFLEXOLOGY IS A COMPLEX massage intervention, typically delivered to the feet. It is the sixth most popular form of complementary therapy in the United Kingdom. ${ }^{1}$ The largest reflexology training provider, the International institute of Reflexology (IIR) $)^{2,3}$ delivers training based on the theory that the soles of the feet represent a proportional scaled map or chart of the human body. In this concept, discreet, individual areas (called reflex points) on the feet are believed to map to individual internal organs of the body, ${ }^{4}$ and when these areas are massaged, using distinct reflexology pressure techniques, the corresponding organs are thought to receive an increase in blood supply. ${ }^{5}$

At this time, it is not clear how reflexology massage applied to distinct areas of the feet could be causally connected with a hemodynamic change and robust empirical evidence that validates the claim of a two-way causal relationship between discrete areas of the feet and the perfusion rates of internal organs is scarce. ${ }^{1,6}$ For an organ to receive a greater supply of blood, local vasodilatation related to changes in local paracrine mediators, e.g., nitric oxide, endothelin, and angiotensin (usually produced by smooth muscle and en- dothelial cells in the vessel wall), would have to occur, ${ }^{7,8}$ and these effects are not typically associated with foot massage. Therefore reflexology researchers face two key methodological challenges that need to be addressed if this biologically implausible treatment-related effect is to be scientifically demonstrated. This article identifies these two challenges and proposes novel solutions to both.

\section{Inconsistent Reflexology Foot Maps}

Given the importance of the claim of a specific two-way connection between points on the feet and the internal organs, there is a surprising lack of consistency of reflex point locations in published reflexology foot maps. There are many different reflexology foot charts available, ranging from maps produced by individual therapist trainers right through to charts or maps published and marketed by larger professional reflexology organizations such as the Association of Reflexologists, ${ }^{9}$ the British Reflexology Association, ${ }^{10}$ and the British School of Reflexology. ${ }^{10}$ The vast majority of maps appear to be based on the original Ingham foot chart, ${ }^{11}$ but many of the organ-related reflex points on subsequent maps appear in inconsistent places, depending on the beliefs or constructs of the map provider. ${ }^{12}$

\footnotetext{
${ }^{1}$ School of Nursing, Midwifery \& Health, University of Stirling, Inverness, United Kingdom.

${ }^{2}$ School of Nursing, Midwifery \& Health, University of Stirling, Stirling, United Kingdom.

${ }^{3}$ Highland Heartbeat Centre, Cardiology Unit, Raigmore Hospital, Inverness, United Kingdom.
} 
This inconsistency of published maps presents a serious methodological research challenge if the intention is to isolate a specific treatment-related hemodynamic effect and create a reproducible reflexology intervention. The majority of existing reflexology studies do not provide details of which reflexology map the intervention is founded upon, which means that some of the studies are, in theory, using different reflexology points on which to base the supposedly same intervention. Clearly, if this is the case, there is a lack of consistency in the intervention approach, which arguably invalidates the validity and generalizability of any findings, whether positive or negative.

\section{Poor Experimental Controls}

Aside from the map inconsistencies, many reflexology studies present "sham" reflexology in the form of foot massage as the placebo or experimental control, yet provide no clear description of how the foot massage treatment is distinguishable from general foot massage. ${ }^{1}$ This is a particularly important distinction to consider within the reflexology theoretical construct because foot massage of the plantar foot area may also unintentionally "treat" organ reflex points during the control treatment. Therefore, it is clear that foot massage does not provide a suitably ineffectual form of control in reflexology research.

Furthermore, the majority of studies deliver the reflexology intervention as a "whole system" complex treatment, making no attempt to control for nonspecific effects in order to isolate any active specific effect. Within this research framework, model validity is more of a priority and conceptually, many aspects of a therapeutic intervention are regarded as inherently culture-specific. ${ }^{13,14}$ This interpretation means that the researchers do not attempt to translate the context of the therapy into the experimental framework of the randomized controlled study design, in order to eliminate any nonspecific components, such as placebo, therapeutic or environmental effects. ${ }^{15}$ However, although the whole systems framework allows for more sensitive evaluation of complex health care interventions, ${ }^{14}$ in the case of the specific claims of reflexology, there are ethical considerations that need to be addressed if the value of reflexology relies predominantly on placebo or nonspecific effects. The key issue here being whether researchers can provide evidence for the specific hemodynamic claims of reflexology in order to justify cost effectiveness for its purchasers. ${ }^{16}$

To date, only two studies have attempted to isolate a specific effect by contrasting massage to a specific area of the foot while measuring the associated organ perfusion rates compared with massage to "nonactive" areas of the feet as the control, ${ }^{17,18}$ but even those two articles failed to identify the organ-associated foot reflexology map location used, which makes it difficult to reproduce their methods.

\section{A Reductionist Solution}

In response to these two distinct challenges, we have devised a potential experimental solution that we believe can address both the map inconsistency problem and enable a standardized reflexology control to be developed. We believe that if both of these methodological challenges are resolved, any specific hemodynamic treatment effect unique to reflexology should be able to experimentally reveal itself. We believe that if both of these methodological challenges are resolved, any specific hemodynamic treatment effect unique to reflexology should therefore be able to experimentally reveal itself.

We propose that reflexology researchers adopt a reductionist foot-map approach, based on the fact that despite considerable variations, all reflexology charts appear to have one consistent overall structural design and it is this. All the reflex points associated with the top half of the body, the brain, brain stem, central nervous system, heart, lungs, kidneys, and liver are consistently located in the top half of the sole of the foot. From now on, this area will be referred to as the "forefoot" area. Whereas, all the reflex points associated with organs and structural and mechanical body parts found below the waist, such as the knee, hip, sciatic area, reproductive/digestive organs, small and large intestine, the ovary/testicles, uterus, and prostate gland, are always found in the bottom half of the sole of the foot. This area will now be referred to as the "heel." Therefore, if a researcher wished to examine the claim of a hemodynamic two-way link between an individual organ and reflex point on the foot, an experimentally reproducible reductionist map strategy can be adopted so that either the forefoot or heel is treated as the active intervention, depending on which half contains the relevant reflex point. ${ }^{16}$ A separate treatment to the other half of the foot could then act as the control.

Using the liver or spleen organs as examples of this strategy, in our reductionist model, regardless of map inconsistencies, the liver and spleen reflex points are consistently located in the forefoot or top half of either foot. Therefore, if the experimental aim was to isolate a specific two-way hemodynamic relationship between treatment to either the liver or spleen point and a hemodynamic change in the status of either organ, a short standardized reflexology treatment protocol could be developed that could be applied in two distinct treatments to both halves of the feet, making reflexology treatment to the top half of the foot (forefoot) act as the liver or spleen organ point intervention (even though the exact organ point locations remains inconsistent in published maps) and the heel treatment act as the control (the liver and spleen organ reflex points are never found in the heel area).

By using the heel treatment as the control, an experimentally inert reflexology comparison can be achieved in which nonspecific effects such as the compassion of the therapist, the treatment environment, the act of lying supine, expectation of the receiver, and the relaxing effects of simple foot massage $\mathrm{e}^{19,20}$ would be equally present in both intervention and control. Therefore if the beat-to-beat hemodynamic status of either the liver or spleen organs were measured throughout the intervention and control treatment, and the experiment used reflexology-naive subjects in a randomized, double-blinded repeated measures study design, it should be possible to isolate and detect a specific treatment-related hemodynamic effect on the organ in question; a specific effect that should only be present at some point during the intervention (revealed by real-time measurement of the organ or organs of interest), quite distinct from nonspecific components that should be equally present in both, thereby resolving both the map inconsistency problem and providing a suitably robust experimental control. 


\section{Conclusion}

This article has proposed a novel reductionist solution to address the methodological challenges of inconsistent reflexology maps and poor experimental controls in reflexology research. We believe that adopting a reductionist approach to the reflexology foot map will help researchers overcome the problem of experimental reproducibility in the face of inconsistent point location and provide an inert control, which for the first time can allow a specific treatment-related two-way hemodynamic effect between reflex points in either half of the foot and their associated hemodynamically measurable organs to experimentally reveal itself.

\section{Acknowledgment}

The authors wish to thank Tracey Smith, Reflexology Support Manager at the Association of Reflexologists.

\section{Author Disclosure Statement}

No financial conflicts exist.

\section{References}

1. Ernst E. Is reflexology an effective intervention? A systematic review of randomised controlled trials. Med J Aust 2009;191:263-265.

2. International Institute of reflexology. Branches of the International Institute of Reflexology. 2012. http://www.reflexology-usa.net/branches.htm Accessed June 27, 2011.

3. International Institute of Reflexology I. The nation's leading authority. 2012. http://www.reflexology-usa.net/branches .htm Accessed February 02, 2011.

4. IIR IIoR. Original Inham Method Foot Wall Chart. 2011. http://store.reflexology-uk.net/products?tag $=$ charts $\% 20 \%$ 26\%20promo Accessed February 06, 2011.

5. Ingham E. The original works of Eunice D Ingham; Stories the Feet Can Tell Thru Reflexology; Stories the Feet Have Told Thru Reflexology. 2nd Revised edn. St. Petersburg, Florida, Ingham Publishing Inc. 1984:3-4.

6. Ernst E, Posadzki P, Lee MS. Reflexology: An update of a systematic review of randomised clinical trials. Maturitas 2011;68:116-120.

7. Duvall WL. Endothelial dysfunction and antioxidants. Mt Sinai J Med 2005;72(2):71-90.

8. Higashi Y, Yoshizumi M. New methods to evaluate endothelial function: Method for assessing endothelial function in humans using a strain-gauge plethysmorgraphy: Nitric oxide dependent and independent vasodilation. J Pharmacol Sci 2003;93:399-404.

9. Association of Reflexologists. Reflexology Chart. 2011. http:// www.reflexology.org /index.php?target $=$ products\& product id =3 Accessed February 09, 2011.
10. British Reflexology Association. Reflexology Foot Chart. 2011. http://www.britreflex.co.uk/product_reflexology_foot_ chart.html Accessed February 02, 2011.

11. Ingham E. Stories the Feet Can Tell Thru Reflexology; Stories the Feet Have Told Thru Reflexology. Ingham Publishing Inc. St. Petersburg, Florida, 1938.

12. Jones J, Thomson P, Lauder W, Leslie SJ. Reported treatment strategies for reflexology in cardiac patients and inconsistencies in the location of the heart reflex point: an online survey. Complementary Therapies in Clinical Practice. 2012 18(3):145-150 Epub 2012 Jun 27.

13. Verhoef MJ, Lewith G, Ritenbaugh C, Boon H, Fleishman S, Leis A. Complementary and alternative medicine whole systems research: Beyond identification of inadequacies of the RCT. Complement Ther Med 2005;13:206-212.

14. MacPherson H. Pragmatic clinical trials. Complement Ther Med 2004;12:136-140.

15. Black C. Assessing complementary practice: Building consensus on appropriate research methods. Report of an independent advisory group. Introduction $x i$, The Kings Fund. http://www.kingsfund.org.uk/sites/files/kf/Assessingcomplementary-practice-Building-consensus-on-appropriateresearch-methods-Dame-Carol-Black-The-Kings-Fund-July2009.pdf Accessed January 04, 2011.

16. Black C. Assessing complementary practice: Building consensus on appropriate research methods. Report of an independent advisory group. Summary ix, The Kings Fund. http://www.kingsfund.org.uk/sites/files/kf/Assessingcomplementary-practice-Building-consensus-on-appropriateresearch-methods-Dame-Carol-Black-The-Kings-Fund-July2009.pdf Accessed January 04, 2011.

17. Sudmeier H, Bodner G, Egger I, Mur E, Ulmer H, Herold M. Changes of renal blood flow during organ-associated foot reflexology measured by colour Doppler sonography. Forsch Komplementarmed 1999;6:129-134. (In German.)

18. Mur E, Schmidseder S, Egger I, et al. Influence of reflex zone therapy of the feet on intestinal blood flow measured by color Doppler sonography. Forsch Komplementarmed Klass Naturheilkd 2001;8:86-89. (In German.)

19. Wang M, Tsai $P$, Lee $P$, Chang W, Yang C. The efficacy of reflexology: Systematic review. J Adv Nurs 2008;62:512-520.

20. Siev-Ner I, Gamus D, Lerner-Geva L, Achiron A. Reflexology treatment relieves symptoms of multiple sclerosis: A randomized controlled study. Mult Scler 2003;9:356-361.

Address correspondence to: Jenny Jones, BSc (Hons), RN School of Nursing, Midwifery $\mathcal{E}$ Health University of Stirling Old Perth Road Inverness, IV2 3JH United Kingdom

E-mail: jenny.jones@stir.ac.uk 